ISSN 0103-5150

Fisioter. Mov., Curitiba, v. 26, n. 1, p. 115-121, jan./mar. 2013 Licenciado sob uma Licença Creative Commons

\title{
Efeito da Kinesio Taping sobre força máxima e resistência de força em padelistas
}

\author{
Effects of Kinesio Taping on maximal and \\ endurance strength in paddle players
}

\section{Marcos Atrib Zanchet ${ }^{[a]}$, Fabrício Boscolo Del Vecchio ${ }^{[b]}$}

[a] Mestre em Educação Física pela Universidade Federal de Pelotas (UFPel), Pelotas, RS - Brasil, e-mail: marcoszanchet@gmail.com

[b] Doutor em Educação Física pela Universidade Estadual de Campinas (Unicamp), docente na Escola Superior de Educação Física da Universidade Federal de Pelotas (UFPel), Pelotas, RS - Brasil, e-mail: fabricio_boscolo@uol.com.br

\section{Resumo}

Introdução: 0 emprego da Kinesio Taping (KT) em competições esportivas, como tentativa de incrementar o rendimento de atletas de diferentes modalidades, tem aumentado substancialmente. No entanto, poucos são os estudos que investigaram os efeitos desse material no desempenho físico. Objetivos: Avaliar os efeitos da KT na força isométrica máxima de preensão manual (FIPM) e na resistência de força (RF) de praticantes de pádel após realização de um set da modalidade. Materiais e métodos: Em um estudo contrabalanceado, de medidas repetidas, 14 indivíduos foram submetidos à mensuração de FIPM e RF em três condições distintas: repouso, após um set utilizando a KT e após um set sem utilizar KT. As variáveis foram medidas no hemicorpo dominante, com dinamômetro eletrônico de preensão manual com sujeitos sentados, cotovelo flexionado a $90^{\circ}$ firmemente apoiado contra o tronco e antebraço em rotação neutra. Para FIPM, foi analisada a média de três medidas e, para RF, realizou-se cronometragem do tempo em que o sujeito sustentava 70\% da FIPM. Resultados: Não foram observadas diferenças significativas entre as três condições quanto a FIPM ( $p=0,86)$. Para RF, encontrou-se diminuição significativa para condição sem KT (24,16 $\pm 12,49$ s) quando comparada ao repouso $(33,46 \pm 14,05$, p = 0,01), o que não ocorreu com o emprego da KT $(31,01 \pm 11,85)$. Conclusões: A aplicação da KT reduziu o decréscimo da RF em praticantes de pádel após um set dessa modalidade, mas não exerceu efeito sobre a FM.

Palavras-chave: Força muscular. Força da mão. Resistência física. Kinesio Taping. Bandagem. 


\section{Abstract}

Introduction:The use of Kinesio Taping (KT) in athletic competitions as an attempt to increase the athletes' performance of different modalities has increased substantially. However, there are few studies assessing the effects of this method on physical performance. Objectives: To evaluate the effects of KT in maximal isometric strength handgrip (FIPM) and the endurance strength (RF) of paddle players after performing one game set. Materials and methods: This counterbalanced study of repeated measures enrolled 14 people who were submitted to FIPM and RF measuring in three distinct conditions: at rest, and after one game set with or without KT. The variables were measured on the dominant side, with an electronic handgrip dynamometer with the subject seated, elbow flexed a $90^{\circ}$ against the trunk and forearm in neutral rotation. For FIPM it was considered the average of three measurements and for RF it was measured the time that the subject was able to sustain $70 \%$ of the FIPM. Results: No significant differences were found between the three conditions regarding FIPM $(p=0.86)$. For $R F$, it was observed a significant reduction on the condition without KT (24.16 $\pm 12.49 s)$ when compared to rest (33.46 $\pm 14.05, p=0.01)$, which did not happen when using KT (31.01 \pm 11.85$)$. Conclusions: The application of KT inhibited the reduction of RF in paddle players after one game set, but it did not cause any effects on FIPM.

Keywords: Muscle strength. Grip strength. Physical endurance. Kinesio Taping. Tape.

\section{Introdução}

O pádel é um esporte popular em diversos países, dentre eles, Argentina, Uruguai, Brasil e Espanha (1). A exemplo do tênis e do squash, o número de seus praticantes é crescente, mesmo sendo considerado relativamente novo no âmbito competitivo (2). Embora semelhante ao tênis em relação às regras e demandas fisiológicas, difere-se por ser sempre praticado em duplas e permitir que as paredes laterais e do fundo da quadra sejam utilizadas no jogo, o que confere mais emoção e dinamismo às disputas dos pontos $(3,4)$.

Apesar do notável aumento no número jogadores dessa modalidade, são poucas as investigações científicas que fornecem informações sobre as demandas fisiológicas necessárias a fim de fundamentar a prescrição dos treinamentos para obter aumento do rendimento esportivo (5). Complementarmente, assim como as demais modalidades competitivas, 0 pádel exige condições técnicas, táticas e físicas específicas para a prática (3). Por se tratar de esporte de raquete, destaca-se a relevância da combinação entre aptidão aeróbia e anaeróbia, com velocidade, potência, agilidade, flexibilidade e força muscular para o êxito competitivo $(6,7)$.

Assumindo-se, de fato, a relevância da força isométrica de preensão manual (FIPM) para realização de gestos da modalidade, como o voleio - golpe mais frequentemente utilizado no pádel (4) -, observa-se o aumento da utilização de Kinesio Taping (KT) com vistas ao aprimoramento agudo dessa variável $(8,9)$ por facilitar a contração muscular (10).

Nesse sentido, Mohammadi et al. (11) utilizaram a KT para modificar de modo agudo os níveis de força de preensão manual de 40 sujeitos e observaram aumentos nos homens e nas mulheres (ambos $\mathrm{p}<0,05$ ) (11). Além disso, Schneider et al. (12) investigaram se ocorreria diminuição da fadiga e manutenção da força muscular após aplicação de KT em 14 tenistas com epicondilite lateral. Foi possível observar redução significante $(p=0,032)$ da força na condição sem bandagem quando comparada à aplicação de KT (12). Neste estudo, visamos verificar os efeitos da KT na FIPM e na resistência de força de praticantes de pádel ao considerarmos a realização de um set da modalidade como agente estressante do sistema neuromuscular.

\section{Materiais e métodos}

Tipo de estudo e caracterização das variáveis

O estudo proposto é de caráter experimental, randomizado e com medidas repetidas $(13,14)$. Como variáveis dependentes, foram eleitas força isométrica máxima de preensão manual e resistência de força; 
e, como independente, a presença ou ausência da KT no antebraço dos sujeitos envolvidos.

\section{Sujeitos}

Para determinação do número de pessoas a serem envolvidas no estudo, o cálculo do tamanho amostral se deu a partir dos dados de Mohammadi et al. (11) - 38,33 $\pm 6,5$ kgf na força máxima de preensão manual na pré-intervenção e 42,4 \pm 7,3 kgf após o emprego da Kinesio Taping. Nesse sentido, considerando poder de $80 \%$ e nível de significância de $5 \%$ em um teste $t$ de Student unilateral, seriam necessários 21 homens para a realização do estudo $(15,16)$. No entanto, adotando como critérios de inclusão prática da modalidade há mais de três meses e ausência de lesão no membro superior dominante nos dois anos que antecederam as avaliações, 19 voluntários que praticam o pádel regularmente na cidade de Pelotas, Rio Grande do Sul, foram elegíveis. Com relação aos procedimentos éticos, o estudo respeitou a Resolução n. 196/96 do Conselho Nacional de Saúde, todos os participantes assinaram Termo de Consentimento Livre e Esclarecido e a pesquisa foi aprovada pelo Comitê de Ética e Pesquisa em Seres Humanos da ESEF/UFPel, sob protocolo n. 008/2011.

Delineamento do estudo

Para a realização do estudo, os jogadores foram abordados em seu local de prática, e a mensuração de força isométrica máxima de preensão manual e resistência de força ocorreu em três condições distintas. Primeiramente, todos os indivíduos foram avaliados na situação de repouso (baseline). Logo após a avaliação inicial, eles foram alocados aleatoriamente em duas condições para, então, atuarem durante um set do jogo. As condições envolviam a presença (KT) ou ausência (NKT) da KT. Todos os sujeitos deveriam realizar as três condições, sendo que entre as condições KT e NKT foi respeitado o intervalo de sete a dez dias (Figura 1).

A bandagem KT usada foi a Kinesio Tex Tape ${ }^{\circledR}$ (Kinesio Holding Company, Albuquerque, NM) de cor preta (Figura 2A). Sua aplicação consistiu em duas tiras na forma de "I": uma sobre os músculos extensores, e outra sobre os flexores de punho do membro dominante (Figura 2B), ambas dispostas segundo a direção origem - inserção, com tensão de 25\% (10). A identificação do comprimento da tira foi individual, já que é determinado pela distância entre origem e inserção dos músculos de cada sujeito. Para isso, a KT foi medida a partir do epicôndilo lateral, para aplicação nos extensores, ou epicôndilo medial, para os flexores, até próximo à articulação do punho (17).

\section{Coleta dos dados}

Os dados demográficos foram coletados na avaliação inicial e registrados em formulários específicos. As mensurações dos níveis de força isométrica máxima de preensão manual e resistência de força foram realizadas no hemicorpo dominante com dinamômetro eletrônico de preensão manual que exibe

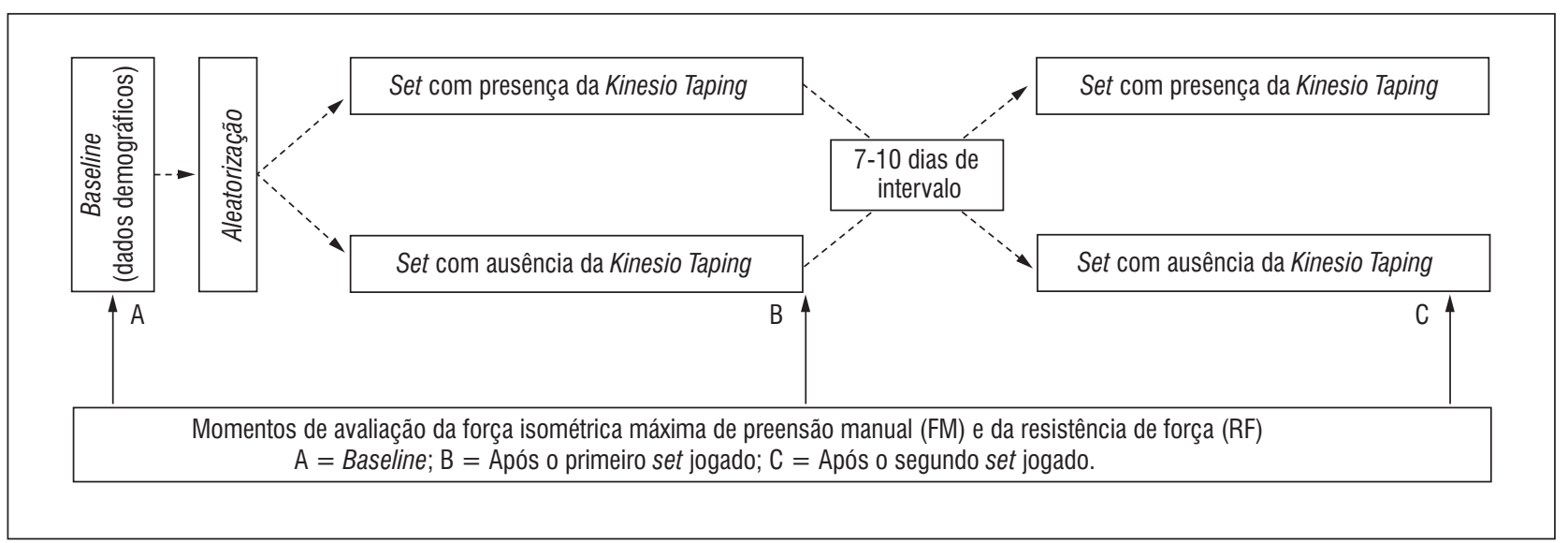

Figura 1 - Delineamento experimental da intervenção

Fonte: Dados da pesquisa. 


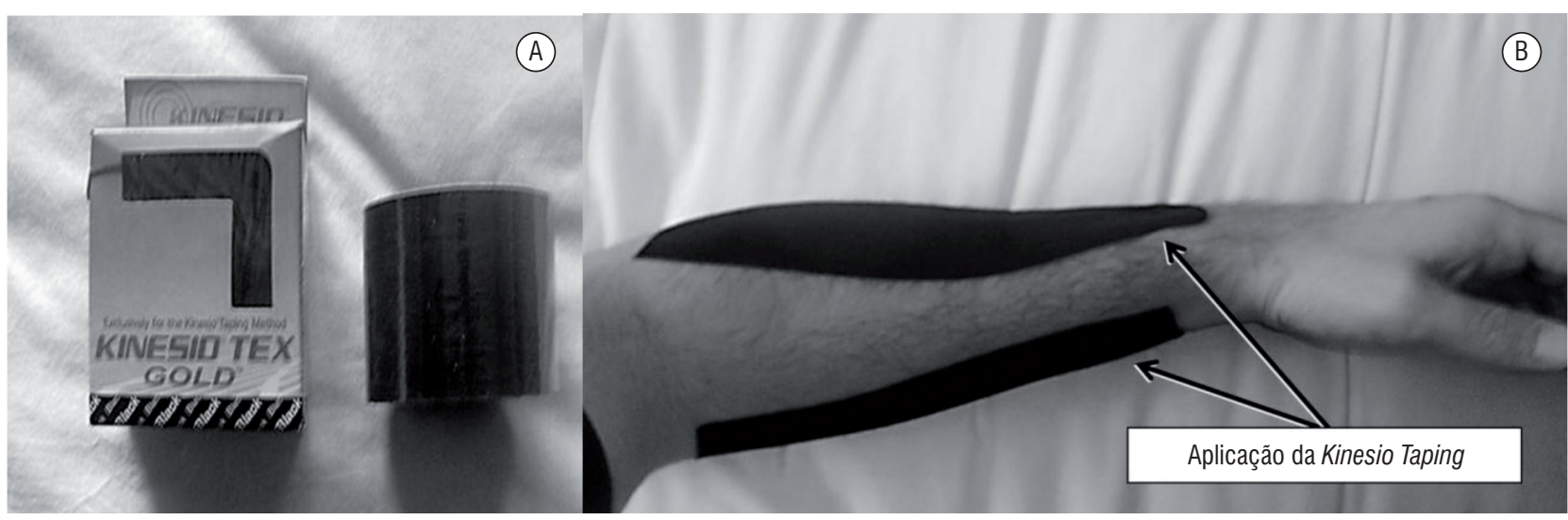

Figura 2 - Bandagem Kinesio Taping (A) e respectivo modo de aplicação em "I" sobre flexores e extensores de punho (B) Fonte: Dados da pesquisa.

coeficiente de correlação intraclasse de 0,9 (Modelo EH 101, DayHome ${ }^{\mathrm{TM}}$ Ltda). Os sujeitos se mantiveram sentados, com o cotovelo firmemente contra o tronco e flexionado a $90^{\circ}$ e com o antebraço em posição de rotação neutra $(18,19)$.

\section{Força isométrica máxima de preensão manual}

Para avaliação da FIPM, os indivíduos pressionaram o dinamômetro com a máxima força possível por cinco segundos e, então, liberaram a pressão da mão (20), com registro do desempenho em display digital. Foram realizadas três medições para cada sujeito com intervalo de um minuto entre elas, e a média dessas medidas foi considerada para análise dos dados. Este procedimento apresenta alta reprodutibilidade, com coeficiente de correlação intraclasse de 0,93 (21).

Resistência de força isométrica máxima de preensão manual

A avaliação da resistência de força foi realizada com protocolo previamente aplicado com praticantes de escalada esportiva (22). Para sua realização, considerou-se $70 \%$ da FIPM obtida no baseline, a qual deveria ser mantida pelo maior tempo possível, com possibilidade de variação de $5 \%$. A identificação da faixa-alvo foi feita por um dos autores e, continuamente, proporcionava feedback ao avaliado. Estudo piloto prévio em nosso laboratório registrou replicabilidade excelente com correlação intraclasse de 0,88 e erro experimental de $0,03(F=15,76 ; p=0,003)$.
Análise dos dados

A análise dos dados foi feita com o software Statistical Package for the Social Sciences (SPSS, versão 14.0 SPSS Inc., Chicago IL). Para apresentação descritiva dos dados, utilizaram-se média e desvio padrão. A distribuição dos dados foi analisada com a prova de Shapiro-Wilk. A comparação da duração dos sets jogados nos dois momentos de avaliação foi realizada com teste $t$ de Student.

Conduziu-se análise de variância de um caminho (ANOVA) com medidas repetidas para testar os diferentes momentos de avaliação (KT, NKT e Baseline), sendo que o teste de Mauchly foi empregado para verificar a esfericidade dos dados e a correção de Greenhouse-Geiser usada quando necessária (13). Identificando-se significância na ANOVA, utilizou-se o teste de Dunnet (com baseline sendo considerado medida controle) para identificação das diferenças (23). 0 nível de significância estatística foi definido em $\mathrm{p}<0,05$.

\section{Resultados}

Dos 19 sujeitos inicialmente incluídos no estudo, cinco não compareceram para as três condições de avaliação e foram excluídos da análise. Participaram 14 indivíduos (13 homens e 1 mulher) com 29,3 \pm 10,8 anos, $1,75 \pm 0,7 \mathrm{~m}$ de estatura, $75,5 \pm 12,5 \mathrm{~kg}$ e tempo de prática do pádel de 6,2 $\pm 6,6$ anos. A frequência semanal de jogo variou entre uma e cinco vezes, sendo que $35,7 \%$ atuam na hemiquadra esquerda, $35,7 \%$ na hemiquadra direita e $28,6 \%$ em ambos os lados. 
Com relação à duração dos sets analisados, não houve diferenças entre os dois momentos de avaliação: respectivamente, $29,14 \pm 6,18$ min e $26,78 \pm$ $9,03 \min (t=0,8 ; p=0,42)$. Quanto à força isométrica máxima de preensão manual, não foram observadas diferenças significativas ( $\mathrm{F}=0,15 ; \mathrm{p}=0,86)$ entre as três condições para (Quadro 1). Oito indivíduos obtiveram aumento da FIPM sem o uso da KT, ao passo que seis pessoas obtiveram diminuição. Na média, o delta percentual de variação foi de $-2,4 \pm 12,4 \%$. Ainda acerca da FIPM com uso da KT, metade da amostra teve o desempenho aumentado, ao passo que a outra metade, não $(0,07 \pm 13,26 \%)$.

Já para resistência de força, encontrou-se diminuição significativa do desempenho na condição NKT quando comparada à baseline $(\mathrm{F}=6,11 ; \mathrm{p}=0,01)$ (Quadro 1). Acerca da resistência de força, sem o uso da KT, apenas duas pessoas expressaram melhora após um set de pádel, sendo que a queda média do grupo foi de $52,6 \pm 50,7 \%$. Com uso da KT, cinco pessoas conseguiram desempenhos superiores à situação baseline, e sete tiveram diminuição. Nessa condição, a queda de desempenho foi de $12,5 \pm 43,2 \%$.

\section{Discussão}

Diversos estudos investigaram o efeito da KT sobre a força muscular em diferentes populações $(8,11,18,19,24,25)$. Essas investigações apresentam resultados conflitantes quanto ao benefício da bandagem sobre essa variável. No mesmo sentido, o objetivo desse estudo foi verificar o efeito da KT sobre FIPM e resistência de força em praticantes de pádel. Nossos resultados mostram que não ocorreu diferença nos níveis de FIPM entre as duas condições durante um único set do jogo. No entanto, menor redução na resistência de força foi verificada quando os indivíduos atuaram utilizando a KT em forma de "I" sobre flexores e extensores de punho.

A menor redução da resistência de força durante a aplicação KT está de acordo com o descrito previamente (12). Observou-se diminuição menos intensa da força dos músculos do antebraço de tenistas quando a KT foi aplicada em forma de "Y" sobre esses músculos. A explicação para isso pode ser atribuída aos mecanismos fisiológicos de ação propostos para KT, como proporcionar estímulo tátil através da pele e ativação/inibição de mecanorreceptores, os quais causam alterações fisiológicas no local da aplicação da bandagem $(8,26)$, melhora da circulação sanguínea (27) e aumento na propriocepção $(28,29)$ e, consequentemente, aumentam a excitabilidade e alteram o padrão de contração muscular $(10,12,19)$.

Dessa forma, a aplicação da KT sobre o antebraço pode se tornar útil para auxiliar na manutenção da força muscular e do desempenho, o que poderia contribuir para resultados competitivos superiores (30). Ao se considerar que a intermitência da modalidade gera fadiga (2) e que esta reduz a acurácia dos golpes, a manutenção da resistência de força pode se constituir como estratégia relevante para a aplicação sucessiva de golpes com a raquete.

Com relação à FIPM, não foram observadas diferenças entre as condições com e sem KT, discordando da afirmação de Kase et al. (10) de que a KT teria

Quadro 1- Efeito da Kinesio Taping sobre a força máxima e resistência de força

\begin{tabular}{lccccc}
\hline Variáveis de interesse & & Média & DP & F & p \\
\hline Força máxima (kgf) & & & 0,15 & 0,86 \\
& Repouso & 40,41 & 10,98 & & \\
& Sem KT & 39,85 & 10,84 & & \\
& Com KT & 40,47 & 9,80 & & $0,01^{*}$ \\
Resistência de força (seg) & & & & \\
& Repouso & 33,46 & 14,05 & & \\
& Sem KT & 24,16 & 12,49 & & \\
& Com KT & 31,01 & 11,85 & & \\
&
\end{tabular}

Legenda: * = diferença significante entre situações repouso e sem aplicação da KT.

Fonte: Dados da pesquisa. 
capacidade de facilitar a contração muscular. Nosso resultado é semelhante ao encontrado por Chang et al. (18), que não observaram diferença nos valores de FIMP entre as condições KT, sem KT e bandagem placebo. Porém, esses autores encontraram melhora na percepção de força para o grupo KT, o que não foi avaliado nesta investigação. É importante salientar que a aplicação da bandagem KT pode afetar as condições psicológicas dos atletas; portanto, a aplicação da KT como forma de placebo se torna interessante para reduzir os efeitos psicológicos da fadiga muscular (18).

Outros estudos também não encontraram benefícios da KT sobre a força muscular $(23,24)$, fato que contrasta com o descrito por Vithoulk et al. (8), que encontraram aumento da força muscular após a aplicação de KT em membros inferiores. Com relação à variável-alvo deste estudo (FIPM), Mohammadi et al. (11) e Lee et al. (19) observaram aumento nos níveis de força após a aplicação de KT. A diferença entre os resultados dessas investigações podem ser decorrentes das formas e tensões aplicadas à bandagem, pois oferecem estímulos táteis de intensidades diferentes. Assim, a tensão aplicada à KT nesta pesquisa $(25 \%)$ pode não ter sido suficientemente forte para alterar os padrões de excitabilidade muscular e aumentar a FIPM.

\section{Limitações do estudo}

Não foi possível controlar algumas variáveis que poderiam interferir nos resultados do estudo, como temperatura e umidade do ambiente, intensidade e duração dos jogos e tempo de recuperação entre os pontos. Nesse contexto, a interferência das variáveis relacionadas ao jogo pode ter sido diminuída a partir do controle de duração dos sets jogados. Estatisticamente, a duração total do esforço, nas duas ocasiões, foi idêntica $(p=0,42)$.

\section{Considerações finais}

Esta pesquisa não registrou diferença estatisticamente significativa quanto à força isométrica máxima de preensão manual após a utilização de KT. No entanto, a aplicação da bandagem em forma de "I" sobre os flexores e extensores de punho reduziu o decréscimo na resistência de força em praticantes de pádel após jogarem um set da modalidade.

\section{Referências}

1. Morlán Luna VM, Sanz Arazuri ES. Promoción del paddle de competición desde las primeras etapas deportivas (6-12 años). Um proyecto de intervención. Retos. 2008;13:46-9.

2. Lora MH, Corrales BS, Páez LC. Demandas fisiológicas de la competición en pádel. Rev Int Cienc Deporte. 2007;3(8):53-8. doi:10.5232/ricyde2007.00805.

3. Corralez BS, Lora MH, Páez LC. Demandas fisiológicas y características estructurales de la competición en pádel masculino. Apunts Educ Fís Deportes. 2008;94:23-8.

4. Roque JIA, Iturriaga FMA. Relación entre las acciones finales con el drive y el revés con el rendimiento en un deporte de raqueta y muro: frontenis olímpico. Apunts Educ Fís Deportes. 2009;96:66-75.

5. Reilly T, Morris T, Whyte G. The specificity of training prescription and physiological assessment: a review. J Sports Sci. 2009;27(6):575-89. doi: 10.1080/ 02640410902729741.

6. Less A. Science and the major racket sports: a review. J Sports Sci. 2003;21(9):707-32. doi:10.1080/ 0264041031000140275.

7. Kovacs MS. Applied physiology of tennis performance. Br J Sports Med. 2006;40(5):381-6. doi:10.1136/ bjsm.2005.023309.

8. Vithoulka I, Beneka A, Agelousis N, Karatsolis K, Diamantopoulos K. The effects of Kinesio Taping on quadriceps strength during isokinetic exercise in healthy non-athlete women. Isokinet Exerc Sci. 2010;18:1-6.

9. Chang HY, Wang CH, Chou KY, Cheng SC. Could forearm Kinesio Taping improve strength, force sense, and pain in baseball pitchers with medial epicondylitis? Clin J Sport Med. 2012;22(4):327-33. doi:10.1097/ JSM.0b013e318254d7cd.

10. Kase K, Wallis J, Kase T. Clinical Therapeutic applications of the Kinesio Taping Method. Tóquio: Ken Ikai Co Ltd.; 2003.

11. Mohammadi KH, Pouretezad M, Shokri E, Tafazoli M, Sioki N. The effect of forearm kinesio taping on hand grip strength of healthy people. J Kerman University of Medical Sciences. 2010;17(3):248-56. 
12. Scheneider M, Rhea M, Bay C. The Effect of kinesio tex tape on muscular strength of the forearm extensors on collegiate tennis athletes. Theratape.com. 2010 [cited 2012 Mai 12]. Available at: http://www. kinesiotaping.com/images/kinesio-association/pdf/ research/2010-3.pdf.

13. Maia J, Garganta R, Seabra A, Lopes V, Prista A, Freitas D. Uma nota didáctica breve no uso esclarecido de procedimentos estatísticos em análise de dados repetidos no tempo. Um estudo guiado para investigadores das Ciências do Desporto. Rev Port Cien Desp. 2004;4(3):115-33.

14. Jones B, Kenward M. Design and analysis of cross-over trials. Boca Raton: CRC Press LLC; 2003.

15. Whitley E, Ball J. Statistics review 4: Sample size calculations. Crit Care. 2002;6(4):335-41. doi:10.1186/ cc1521.

16. Ayres M, Ayres M Júnior, Ayres DL, Santos A, Ayres LL. BIOESTAT: aplicações estatísticas nas áreas das ciências bio-médicas. Belém: Fundação Mamirauá; 2005.

17. Lippert L. Clinical Kinesiology for physical therapist assistants. Philadelphia: FA. Davis Company; 2000.

18. Chang HY, Chou KY, Lin JJ, Lin CF, Wang CH. Immediate effect of forearm Kinesio taping on maximal grip strength and force sense in healthy collegiate athletes. Phys Ther Sport. 2010;11(4):122:7.

19. Lee JH, Yoo WG, Lee KS. Effects of Head-neck rotation and Kinesio Taping of the Flexor Muscle on Dominant hand Grip Strength. J PhysTher Sci. 2010;22:285-9.

20. Brown L, Weir J. ASEP Procedures Recommendations I. Accurate assessment of muscular strength and power. J Exerc Physiol. 2010 [cited 2012 Mai 5]. Available at: http://faculty.css.edu/tboone2/asep/Brown2.pdf.

21. Kroll W. Reliability of a selected measure of human strength. Res Q Exerc Sport. 1962;33:410-7.

22. Watts P, Newbury V, Sulentic J. Acute changes in handgrip strength, endurance and blood lactate with sustained sport rock climbing. J Sports Med Phys Fit. 1996;36(4):255-60.

23. Field A. Descobrindo a estatística usando o SPSS. Porto Alegre: Artmed; 2009.
24. Fu T, Wong AMK, Pei YC, Wu KP, Chou SW, Lin YC. Effect of Kinesio taping on muscle strength in athletes - a pilot study. J Sci Med Sport. 2008;11:198-201. doi:10.1016/j.jsams.2007.02.011.

25. Rodriguez-Moya A, González-Sánchez M, Cuesta-Vargas AI. Efecto del vendaje neuromuscular a corto plazo en la fuerza en la extensión de rodilla. Fisioterapia. 2001;33(6):256-61.doi:10.1016/j.ft.2011.07.007.

26. Yoshida A, Kahanov L. The effect of kinesio taping on lower trunk range of motions. Res Sports Med. 2007;15:103-12. doi:10.1080/15438620701405206.

27. Marban RM, Rodrigues EF, Navarrete PI, Vega DM. The effect of Kinesio taping on calf's injuries prevention in triathletes during competition. Pilot experience. J Hum Sport Exerc. 2011;6:305-8. doi:10.4100/ jhse.2011.62.10.

28. Yasukawa A, Patel P, Sisung C. Pilot study: investigating the effects of kinesio taping in acute pediatric rehabilitation setting. Am J Occup Ther. 2006;60:104-10. doi:10.5014/ajot.60.1.104.

29. Ribeiro MO, Rahal RO, Kakanj AS, Bittar DP. O uso da bandagem elástica Kinesio no controle da sialorréia em crianças com paralisia cerebral. Acta Fisiatr. 2009;16(4):168-72. doi:10.1589/jpts.22.285.

30. Khelaf K. Rôle et place des bandages adhésifs (tape) actifs de couleurs: application pratique dans la pathologie du conflit de la coiffe des rotateurs. Kinesither Rev. 2010;10(104-105):29-33.

Recebido: 01/08/2012

Received: 08/01/2012

Aprovado: 05/01/2013

Approved: 01/05/2013 